\title{
Hemolytic Disease of the Fetus and Newborn due to Intravenous Drug Use
}

\author{
Kara B. Markham, MD ${ }^{1}$ Scott R. Scrape, MD ${ }^{2}$ Mona Prasad, DO ${ }^{1} \quad$ Karen Q. Rossi, RN ${ }^{3}$ \\ Richard W. O'Shaughnessy, MD ${ }^{1}$
}

\author{
${ }^{1}$ Department of Obstetrics and Gynecology, The Ohio State University \\ Wexner Medical Center, Columbus, Ohio \\ 2 Department of Pathology, The Ohio State University Wexner Medical \\ Center, Columbus, Ohio \\ ${ }^{3}$ Department of Obstetrics and Gynecology, Mount Carmel Health \\ System, Columbus, Ohio
}

Am J Perinatol Rep 2016;6:e129-e132.

\author{
Address for correspondence Kara B. Markham, MD, Department of \\ Obstetrics and Gynecology, The Ohio State University Wexner Medical \\ Center, 395 W 12th Ave, 5th Floor/Columbus, OH 43210 \\ (e-mail: kara.markham@osumc.edu).
}

\begin{abstract}
Keywords

- hemolytic disease

- fetus

- newborn

- red blood cell

- alloimmunization

- intravenous drug use

- opiate abuse

Objectives The objective is to present a pregnancy complication associated with intravenous drug use, namely, that of red blood cell alloimmunization and hemolytic disease of the fetus and newborn.

Methods An observational case series is presented including women with red blood cell alloimmunization most likely secondary to intravenous drug abuse

Results Five pregnancies were identified that were complicated by red blood cell alloimmunization and significant hemolytic disease of the fetus and newborn, necessitating intrauterine transfusion, an indicated preterm birth, or neonatal therapy.

Conclusions As opioid abuse continues to increase in the United States, clinicians should be aware of the potential for alloimmunization to red blood cell antibodies as yet another negative outcome from intravenous drug abuse.
\end{abstract}

Opiate abuse is an epidemic in the United States. Drug overdose death rates have skyrocketed in the last decade, with death rates due to prescription opioid pain relievers quadrupling between 1999 and 2010. ${ }^{1}$ This same time period also showed a nearly twofold increased risk in deaths due to heroin, a rate that then doubled again between 2010 and 2012. ${ }^{1}$ Data regarding use are more difficult to obtain as such information usually relies upon self-report. In pregnancy, death due to illicit substances is relatively uncommon, but $\sim 4.4 \%$ of women report use of nonmedicinal drugs, with opioids noted to be the second most commonly abused substance. ${ }^{2,3}$ Much of this use is initiated as abuse of nonmedically indicated prescription pain pills, but heroin abuse is increasing in the setting of increased legislation limiting opiate prescriptions. Accordingly, the incidence of neonatal abstinence syndrome increased from 1.20 to 3.39 per 1,000 hospital births per year from 2000 to $2009 .^{4}$

Beyond the risk of neonatal abstinence syndrome, there are several other complications and comorbidities associated with opioid use in pregnancy. Preterm birth, poor fetal growth, and intrauterine fetal demise are more common in the setting of opioid abuse, and the risks of withdrawal from narcotics have been well documented. ${ }^{5}$ Drug abuse is also commonly associated with mental health disease, social problems, and financial issues. Beyond this, hepatitis C, hepatitis $B$, and human immunodeficiency virus (HIV) are commonly encountered in this population due to intravenous drug use and needle sharing. We present here another pregnancy complication associated with intravenous drug use, namely, that of red blood cell alloimmunization and hemolytic disease of the fetus and newborn (HDFN).

\section{Methods and Materials}

We present an observational case series of patients with red blood cell alloimmunization and a history of intravenous drug use identified at the Ohio State University (OSU) Wexner Medical Center between 2011 and December 1, 2015. Permission to retain and review patients' data was obtained from received

December 21, 2015 accepted after revision January 21, 2016
DOI http://dx.doi.org/ 10.1055/s-0036-1579646. ISSN 2157-6998.
Copyright (c) 2016 by Thieme Medical Publishers, Inc., 333 Seventh Avenue, New York, NY 10001, USA. Tel: +1(212) 584-4662.
License terms

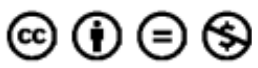


the Ohio State University Biomedical Institutional Review Board before proceeding with this study. All patients denied having red blood cell antibodies in prior pregnancies or any previous children diagnosed with HDFN. Furthermore, each patient reported receiving all medically indicated $\mathrm{Rh}$ immune globulin (RhIG) injections during prior pregnancies, and they all denied a history of blood transfusions.

All laboratory testing was performed at The Ohio State University Medical Center using guidelines established by the American Association of Blood Banks. ${ }^{6}$ An antibody titer of $\geq 1: 32$ at our institution is considered to be critical for most antibodies. Collected data included basic demographic information, maternal medical history (including infections with hepatitis C, hepatitis B, and/or HIV), maternal obstetric history, and indirect antiglobulin test results (antibody screening and titers). Pregnancy outcomes were collected including middle cerebral artery (MCA) Doppler results, specifics regarding intrauterine transfusions or other interventions for HDFN, and gestational age at delivery. Neonatal outcomes were obtained as well, including blood typing, red blood cell antigen status, and cord blood direct antiglobulin test results.

\section{Results}

Selected demographic data and pregnancy outcomes are outlined in - Table 1. We have included patients who required in utero therapy, preterm birth due to concerns about fetal anemia, or neonatal therapy. Two additional cases were identified with low antibody titers (anti-C in one patient and anti-D in the other patient) and no diagnosis of HDFN.

\section{Case 1}

Patient 1 was a 26-year-old G4P2012 who was found to have anti-D and anti-C antibodies with an antihuman globulin titer of $1: 32$ at $9^{0 / 7}$ weeks of gestation. The father of the baby was not available for blood type screening, but determination of fetal $\mathrm{Rh}$ (D) status by maternal plasma cell-free fetal DNA analysis revealed that the fetus was $\mathrm{Rh}+$. The patient underwent fetal MCA Doppler velocimetry every 2 weeks starting at $18^{5 / 7}$ weeks, with normal peak systolic velocities (PSVs) documented until $32^{6 / 7}$ weeks when the fetus developed polyhydramnios, an increased liver length, and an increased heart size with abnormal MCA Doppler assessment ((PSV of $76.87 \mathrm{~cm} / \mathrm{s}$ consistent with 1.68 multiples of the median (MoM)). The patient was counseled at length and declined cordocentesis and intrauterine transfusion. She ultimately received antenatal corticosteroids and underwent a cesarean delivery for repetitive late fetal heart rate decelerations, delivering a 1,995-g male neonate. The neonate had an initial hemoglobin level of $10.0 \mathrm{~g} / \mathrm{dL}$ at the time of delivery and developed significant hyperbilirubinemia soon thereafter. He underwent two exchange transfusions and received intravenous immunoglobulin (Carimune 6\%) therapy three times during his hospital course.

\section{Case 2}

Patient 2 was a 24-year-old G2P0101 who presented at our institution at $29^{0 / 7}$ weeks with hydrops fetalis secondary to alloimmunization to $\mathrm{Rh}(\mathrm{D})$ red blood cell antigens. Her obstetric

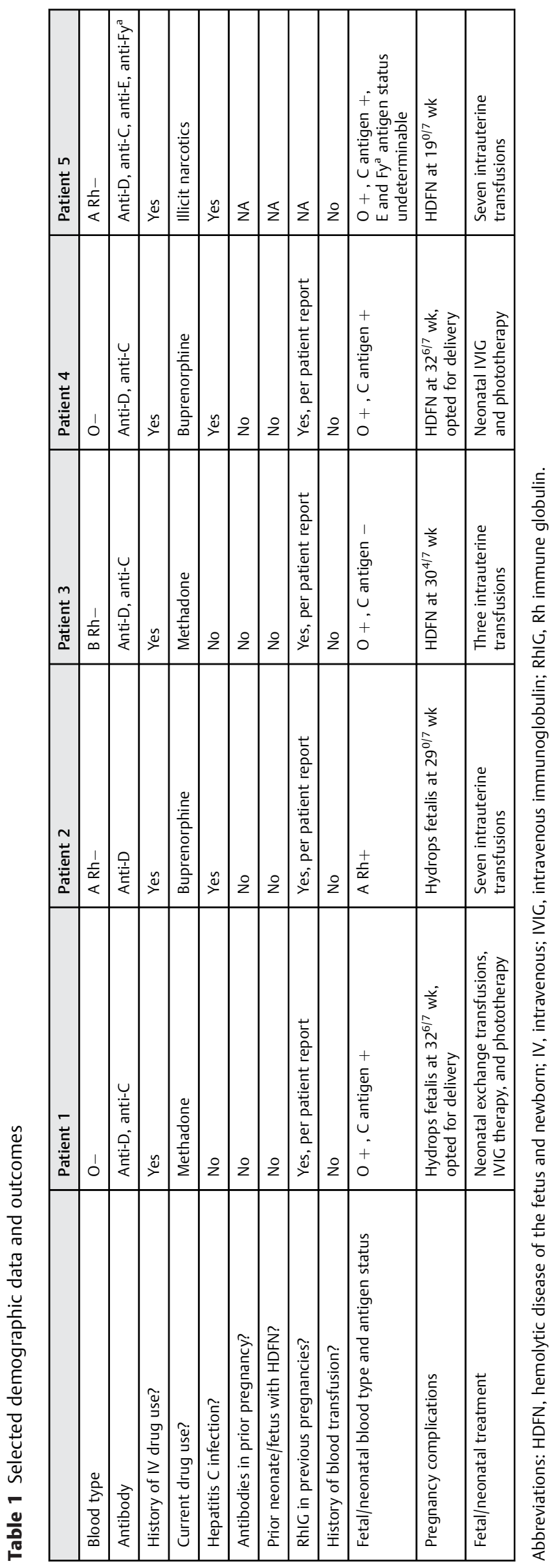


history included a prior vaginal delivery at 30 weeks of gestation of a 1,588-g male neonate with documented O-negative blood type. She was initially found to have anti-D antibodies in the first trimester of the current pregnancy, although antibody titers were not obtained. Per the patient's report, she had an ultrasound approximately 1 month prior to evaluation at OSU, at which time no concerns were noted. She then had an ultrasound 9 days prior to transfer to our hospital, with visualization of fetal ascites. A follow-up ultrasound 7 days later revealed worsening fetal ascites with interval development of fetal scalp edema, prompting transfer to OSU. On arrival at our institution, the presence of anti-D antibodies was confirmed with an antihuman globulin titer of 1:512. Ultrasound here showed fetal ascites, a small pericardial effusion, diffuse skin edema, and elevated PSVs on MCA Doppler evaluation (bedside ultrasound, actual PSV values not recorded), consistent with hydrops fetalis secondary to HDFN. The patient underwent cordocentesis on hospital day \#2 showing an initial hemoglobin level of $2.8 \mathrm{~g} / \mathrm{dL}$ with subsequent intrauterine transfusion of red blood cells resulting in a posttransfusion hemoglobin level of $5.9 \mathrm{~g} / \mathrm{dL}$. During that hospitalization, she underwent additional intrauterine transfusions on hospital days \#3, \#6, \#9, and \#16. Prior to the fifth transfusion, the fetal skin edema was noted to have resolved and afterwards a hemoglobin level of $15.4 \mathrm{~g} / \mathrm{dL}$ was achieved. Given the improved clinical picture, she was discharged to home at $31^{1 / 7}$ weeks of gestation, but she was seen for additional intrauterine transfusions at $33^{2 / 7}$ weeks of gestation and $35^{5 / 7}$ weeks of gestation. She ultimately delivered a $5 \# 10$-oz neonate at $37^{4 / 7}$ weeks of gestation following spontaneous rupture of membranes. Details regarding this delivery and the neonatal hospital course are not known because the patient delivered at another hospital.

\section{Case 3}

Patient 3 was a 24-year-old G3P2002 who was found to have anti-D and anti-C antibodies with an antihuman globulin titer of $1: 16$ at 13 weeks of gestation. The father of the baby was not available for blood type screening, but determination of fetal Rh (D) status by maternal plasma cell-free fetal DNA analysis was inconclusive on two occasions. Her antibody levels increased to an antihuman globulin titer of 1:64 at $23^{3 / 7}$ weeks of gestation. The patient underwent fetal MCA Doppler velocimetry every week starting at $24^{1 / 7}$ weeks, with normal PSVs documented until $30^{4 / 7}$ weeks at which time the fetus developed abnormal MCA Doppler assessment (PSV of $67.3 \mathrm{~cm} / \mathrm{s}$ consistent with $1.64 \mathrm{MoM}$ ). Given the concerns for HDFN, the patient underwent cordocentesis showing an initial hemoglobin level of $3.7 \mathrm{~g} / \mathrm{dL}$ with subsequent intrauterine transfusion of red blood cells resulting in a posttransfusion hemoglobin level of $11.0 \mathrm{~g} / \mathrm{dL}$. Two additional transfusions were performed at $31^{4 / 7}$ weeks of gestation and $32^{5 / 7}$ weeks of gestation. We planned on another transfusion at $34^{4 / 7}$ weeks of gestation, but the patient was found to have significant oligohydramnios. She was counseled at length at that time and opted to undergo delivery. She subsequently underwent a repeat cesarean delivery at $34^{5 / 7}$ weeks of gestation, delivering a 2,800-g female neonate. The initial neonatal hemoglobin level was $13.4 \mathrm{~g} / \mathrm{dL}$, and no additional transfusions were required during her hospital course

\section{Case 4}

Patient 4 was a 30-year-old G4P3003 who was found to have anti-D and anti-C antibodies with an antihuman globulin titer of $1: 64$ at $12^{2 / 7}$ weeks of gestation. Of note, although the patient had documented hepatitis $C$ with viremia, she reported access to a sterile needle supply and denied a history of needle sharing. The father of the baby was not available for blood type screening, but determination of fetal $\mathrm{Rh}(\mathrm{D})$ status by maternal plasma cellfree fetal DNA analysis revealed that the fetus was $\mathrm{Rh}+$. The patient underwent fetal MCA Doppler velocimetry every 2 weeks starting at $18^{0 / 7}$ weeks, with normal PSVs documented until $32^{1 / 7}$ weeks at which time the fetus developed abnormal MCA Doppler assessment (PSV of $77.6 \mathrm{~cm} / \mathrm{s}$ consistent with $1.75 \mathrm{MoM}$ ). She was counseled at that time about the theoretic concerns for hepatitis $C$ vertical transmission with cordocentesis and intrauterine transfusion, ultimately opting for delivery after antenatal corticosteroid administration. She delivered vaginally at $32^{6 / 7}$ weeks, delivering a $1,860-\mathrm{g}$ female neonate. The initial neonatal hemoglobin level was $12.0 \mathrm{~g} / \mathrm{dL}$. The baby received two doses of intravenous immunoglobulin (Carimune 6\%) therapy and phototherapy for hyperbilirubinemia. The hemoglobin level nadired at $7.8 \mathrm{~g} / \mathrm{dL}$ but then stabilized such that no transfusions were required during her hospital course

\section{Case 5}

Patient 5 is a 22-year-old G1P0 who was found to have multiple red blood cell antibodies with antihuman globulin titers as noted at $11^{6 / 7}$ weeks of gestation: anti-D, 1:512; anti-C, 1:16; anti-E, 1:32; and Anti-Fy ${ }^{\mathrm{a}}, 1: 16$. The father of the baby was not available for blood type screening, but determination of fetal $\mathrm{Rh}(\mathrm{D})$ status by maternal plasma cell-free fetal DNA analysis revealed that the fetus was $\mathrm{Rh}+$. The patient underwent a fetal MCA Doppler velocimetry assessment at $19^{0 / 7}$ weeks revealing an elevated PSV of $51 \mathrm{~cm} / \mathrm{s}$ consistent with $>2$ MoM. Given the concerns for HDFN, the patient underwent cordocentesis showing an initial hemoglobin level of $4.4 \mathrm{~g} / \mathrm{dL}$ with subsequent intrauterine transfusion of red blood cells resulting in a posttransfusion hemoglobin level of $13.4 \mathrm{~g} / \mathrm{dL}$. She received six additional transfusions during the pregnancy, the last of which was performed at $29^{5 / 7}$ weeks and was complicated by persistent fetal bradycardia necessitating delivery via an urgent cesarean delivery. She delivered a 985-g female neonate with a birth hemoglobin level of $11.4 \mathrm{~g} / \mathrm{dL}$. The neonate required phototherapy for hyperbilirubinemia and received two doses of intravenous immunoglobulin (Carimune 6\%) therapy. The neonatal hemoglobin level nadired at $5.6 \mathrm{~g} / \mathrm{dL}$ at 1 month of life, and the baby ultimately received three red blood cell transfusions in the first 35 days of life.

\section{Discussion}

We present here a pregnancy complication owing to intravenous drug use, specifically the occurrence of red blood cell alloimmunization and HDFN. This is a potentially serious complication with implications for both the patient and the fetus/neonate. In the only other reported case series of four women (five pregnancies) with alloimmunization due to needle sharing, outcomes were very poor, with hydrops 
fetalis identified in four of the five fetuses and only two of the five babies surviving to delivery. ${ }^{7}$ With up-to-date monitoring and treatment, however, we obtained excellent outcomes in this population. The patients presented here developed significant HDFN, requiring intrauterine transfusion, an indicated preterm birth, and/or neonatal therapy, but they all delivered a live-born, healthy neonate.

An injection of as little as 0.5 to $1.0 \mathrm{~mL}$ of D-positive red blood cells will cause antibody formation in 50\% of Rh-negative recipients. ${ }^{8}$ In fact, the majority of cases of alloimmunization in pregnancy occur due to fetomaternal hemorrhage of less than $0.1 \mathrm{~mL} .{ }^{9}$ It has been estimated that $41 \%$ of intravenous drug users report sharing needles and/or paraphernalia, ${ }^{10}$ and the state of Ohio currently does not endorse a formal needle-exchange program.

Red blood cell alloimmunization has greatly decreased since the introduction of RhIG as part of routine prenatal/ postpartum care. The occurrence of alloimmunization to the D antigen decreases from $16 \%$ in untreated women after two pregnancies to $0.1 \%$ with appropriate use of RhIG in Rh-negative patients with incompatible fetuses. ${ }^{9}$ Patients exposed to incompatible red blood cell antigens via intravenous drug abuse obviously do not benefit from this preventative measure.

In this case series, we excluded all patients who could have been alloimmunized in another manner, although the major weakness of this case series is that we cannot completely eliminate other causes for sensitization. This issue is particularly applicable as this patient population may not always report an accurate history. For example, patient 3 denied a history of sharing needles, but her hepatitis $\mathrm{C}$ infection suggested otherwise.

Not only is intravenous drug use a previously unreported mechanism for HDFN, but also this patient population presents several other issues that must be considered when deciding on treatment options. First of all, patients with a history of opiate abuse frequently have financial and social issues, factors that can greatly impact their ability to comply with recommendations for frequent visits. For example, these patients may even be incarcerated during their pregnancy. Second, anesthetic options may be complicated at the time of procedures such as cordocentesis or intrauterine transfusion due to patients' altered perception of pain and resistance to the effects of opioid pain relievers. Finally, hepatitis C, hepatitis $B$, and/or HIV infections are relatively common in this population. While such infections are not contraindications to performing an amniocentesis, cordocentesis, or intrauterine transfusion, there are case reports of vertical transmission attributed to such procedures. ${ }^{11,12}$ Infected patients must be counseled about this risk. With development of concerns for HDFN at an early gestational age (i.e., $<32-34$ weeks of gestation), the benefits of intrauterine transfusion and prolonged gestation likely outweigh the risk of maternal-to-child transmission, but delivery may be a more appropriate intervention as the gestational age increases.
Screening for substance abuse is recommended as part of routine prenatal care, and antibody screening is included in routine prenatal laboratory testing. While no specific additions to care are recommended based on the information presented here, clinicians should be aware of the potential for alloimmunization to red blood cell antibodies as yet another negative outcome from intravenous drug abuse.

\section{Conflict of Interest}

The authors have no conflicts of interest to report.

\section{Synopsis}

Red blood cell alloimmunization and HDFN can occur due to intravenous drug abuse.

\section{References}

1 Rudd RA, Paulozzi LJ, Bauer MJ, et al; Centers for Disease Control and Prevention (CDC). Increases in heroin overdose deaths - 28 states, 2010 to 2012. MMWR Morb Mortal Wkly Rep 2014;63(39): 849-854

2 Substance Abuse and Mental Health Services Administration. Results from the 2010 National Survey on Drug Use and Health: summary of national findings. NSDUH Series H-41, HHS Publication No. (SMA) 11-4658. Rockville, MD: SAHMSA; 2011

3 Substance Abuse and Mental Health Services Administration. Results from the 2013 National Survey on Drug Use and Health: summary of national findings. NSDUH Series H-48, HHS Publication No. (SMA) 14-4863. Rockville, MD: SAHMSA; 2014

4 Patrick SW, Schumacher RE, Benneyworth BD, Krans EE, McAllister JM, Davis MM. Neonatal abstinence syndrome and associated health care expenditures: United States, 2000-2009. JAMA 2012; 307(18):1934-1940

5 Whiteman VE, Salemi JL, Mogos MF, Cain MA, Aliyu MH, Salihu HM. Maternal opioid drug use during pregnancy and its impact on perinatal morbidity, mortality, and the costs of medical care in the United States. J Pregnancy 2014;2014:906723

6 Fong MK, Grossman BJ, Hillyer C, Westhoff CM; American Association of Blood Banks. Technical Manual of the American Association of Blood Banks. 18th ed, 2014

7 Bowman J, Harman C, Manning F, Menticoglou S, Pollock J. Intravenous drug abuse causes Rh immunization. Vox Sang 1991;61(2):96-98

8 Klein HG, Anstee DJ, eds. The Rh blood group system (including LW and RHAG). In: Mollison's Blood Transfusion in Clinical Medicine. 12th ed. Oxford, UK: John Wiley \& Sons; 2014:167-213

9 Bowman JM. The prevention of Rh immunization. Transfus Med Rev 1988;2(3):129-150

10 Tassiopoulos K, Bernstein J, Bernstein E. Age and sharing of needle injection equipment in a cohort of Massachusetts injection drug users: an observational study. Addict Sci Clin Pract 2013;8:20-28

11 Giorlandino C, Bilancioni E, D’Alessio P, Muzii L. Risk of iatrogenic fetal infection at prenatal diagnosis. Lancet 1994;343(8902):922-923

12 Minola E, Maccabruni A, Pacati I, Martinetti M. Amniocentesis as a possible risk factor for mother-to-infant transmission of hepatitis C virus. Hepatology 2001;33(5):1341-1342 